\title{
Synthesis and Telomeric G-Quadruplex-Stabilizing Ability of Macrocyclic Hexaoxazoles Bearing Three Side Chains
}

\author{
Yue Ma ${ }^{1}$, Keisuke Iida ${ }^{2}$, Shogo Sasaki ${ }^{1}$, Takatsugu Hirokawa ${ }^{3,4,5}$, Brahim Heddi ${ }^{6,7}$, \\ Anh Tuân Phan ${ }^{7}$ and Kazuo Nagasawa ${ }^{1, *(D)}$ \\ 1 Department of Biotechnology and Life Science, Tokyo University of Agriculture and Technology, \\ 2-24-16 Naka-cho, Koganei, Tokyo 184-8588, Japan; yue-ma@m2.tuat.ac.jp (Y.M.); \\ s319964u@st.go.tuat.ac.jp (S.S.) \\ 2 Department of Chemistry, Chiba University, 1-33 Yayoi, Inage, Chiba 263-8522, Japan; kiida@chiba-u.jp \\ 3 Transborder Medical Reserch Center, University of Tsukuba, 1-1-1 Tennodai, Tsukuba 305-8575, Japan; \\ t-hirokawa@aist.go.jp \\ 4 Division of Biomedical Science, University of Tsukuba, 1-1-1 Tennodai, Tsukuba 305-8575, Japan \\ 5 Molecular Profiling Research Center for Drug Discovery, National Institute of Advanced Industrial Science \\ and Technology, 2-4-7 Aomi, Koto-ward, Tokyo 135-0064, Japan \\ 6 Laboratoire de Biologie et Pharmacologie appliquée, CNRS UMR 8113, ENS paris-saclay 60 avenue du \\ president Wilson, 94230 Cachan, France; brahim.heddi@ens-cachan.fr \\ 7 Division of Physics and Applied Physics, Nanyang Technological University, Singapore 637371, Singapore; \\ PhanTuan@ntu.edu.sg \\ * Correspondence: knaga@cc.tuat.ac.jp; Tel.: +81-42-388-7295
}

Academic Editor: Danzhou Yang

Received: 13 December 2018; Accepted: 10 January 2019; Published: 11 January 2019

\begin{abstract}
G-quadruplexes (G4s), which are structures formed in guanine-rich regions of DNA, are involved in a variety of significant biological functions, and therefore "sequence-dependent" selective G4-stabilizing agents are required as tools to investigate and modulate these functions. Here, we describe the synthesis of a new series of macrocyclic hexaoxazole-type G4 ligand (6OTD) bearing three side chains. One of these ligands, $\mathbf{5 b}$, stabilizes telomeric G4 preferentially over the G4-forming DNA sequences of $c$-kit and K-ras, due to the interaction of its piperazinylalkyl side chain with the groove of telomeric G4.
\end{abstract}

Keywords: macrocyclic oxazole; G-quadruplex; telomere; telomestatin

\section{Introduction}

G-quadruplex (G4) is one of the characteristic higher-order structures of non-B DNAs [1]. G4-forming DNA sequences have been found in telomeres, CpG islands, and gene-promoter regions [2-7], and play critical roles in many biological processes, including replication [8,9], gene transcription [10], and translation [11-13]. Thus, compounds that selectively stabilize G4s are promising tools for investigating those functions, as well as candidate therapeutic agents for diseases related to the G4s. Since the G4 structure consists of two or three G-quartet planes with grooves, most G4 ligands so far reported have targeted the G-quartet or the grooves [14,15]. For example, the G4 ligands TMPyP4 [16,17], PhenDC3 [18], and pyridostatin [14,19] interact with the G-quartet in G4, whereas TOxaPy [20] and distamycin A [21] recognize the groove in telomeric G4.

We have developed a series of macrocyclic hexaoxazoles, called 6OTD [22], as G4 ligands based upon the structure of the natural G4 ligand telomestatin [23,24]. Among these derivatives, we have recently shown that 4,2-L2H2-6OTD [25] and L2G2-2M2EG-6OTD [26], obtained by altering the 
connectivity of the oxazoles or by introducing side chains into the 6OTD core structure, interact selectively with anti-parallel and parallel-type topologies, respectively. Here, we describe the synthesis of a new series of 6OTD derivatives bearing side chains that recognize both the G-quartet and the grooves of G4. One of these ligands, $\mathbf{5 b}$, stabilizes telomeric G4 preferentially over the G4-forming DNA sequences of $c-k i t$ and $K$-ras, due to the interaction of a piperazinylalkyl side chain with the groove of telomeric G4 [27-31]. Selective stabilization of telomeric G4 would lead to the senescence and apoptosis of cancer cells through dissociation of telomeric protective proteins such as TRF2 and POT1 from telomeric DNA [32-36].

\section{Results and Discussion}

\subsection{Design of 6OTD Derivatives for Targeting Telomeric G4}

G-quadruplex structures form a variety of topologies depending upon their sequences, and a promising strategy to design "sequence-dependent" selective ligands [37-39] is to target both the G-quartet and the G4 grooves. We previously analyzed the interaction mode of L2H2-6M(2)OTD $\mathbf{1}$ with telomeric DNA by NMR, which is shown in Figure 1a [40], and found that the core structure of the macrocyclic moiety in 6OTD 1 interacts with the G-quartet of telomeric G4 through a $\pi-\pi$ interaction. In addition, the two aminoalkyl side chains in $\mathbf{1}$ interact with the phosphate backbone of DNA. Notably, the architecture, as shown in Figure 1a, includes an additional potential ligand-binding site, i.e., the groove circled in blue. Thus, based on the structure depicted in Figure 1b, we designed tri-substituted 6OTD 2, which has an additional side chain at C5 of L2H2-6M(2)OTD 1. We expected that this modification would increase the affinity for telomeric $\mathrm{G} 4$ and the specificity of the interaction. Specifically, we designed three types of novel tri-substituted L2H2-6OTD derivatives 3-5, bearing an aminoethyl group, an aminoethoxyethyl group, and a piperazinyethoxyethyl group at $\mathrm{C} 5$ in 2, respectively, and examined their specific interactions with telomeric DNA, as shown in Figure 2.

a)
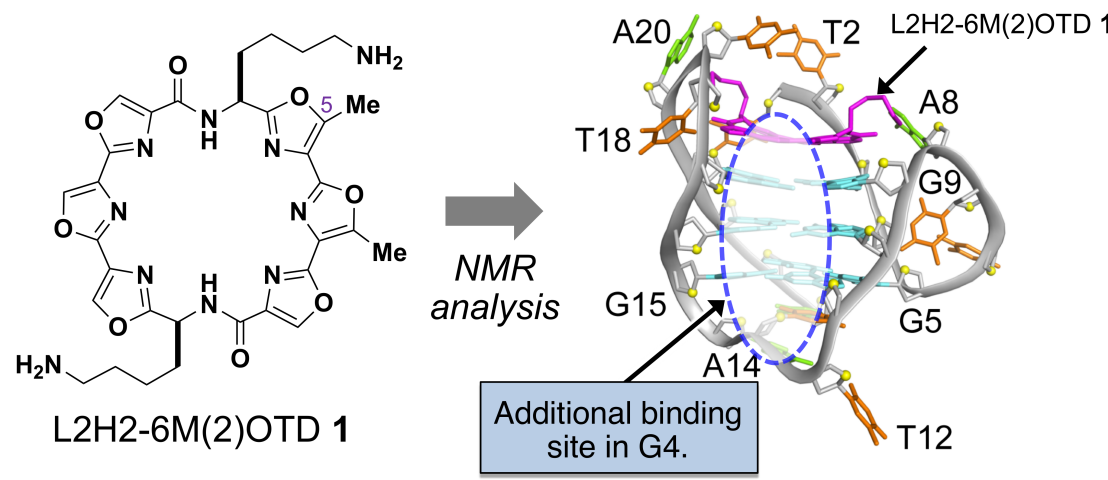

b)

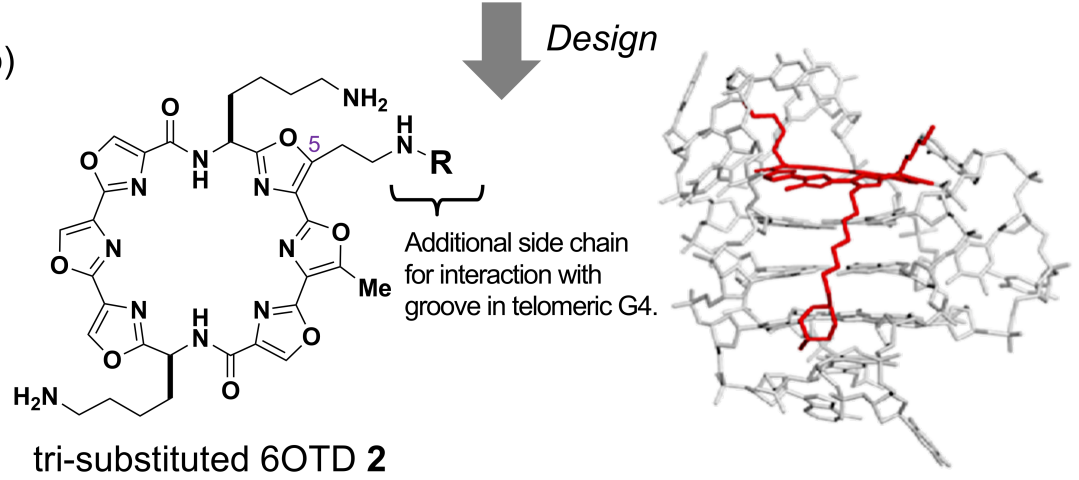

Figure 1. (a) Structure of L2H2-6M(2)OTD 1 and its interaction with telomeric G-quadruplex (G4), as elucidated by NMR analysis; (b) Structure of tri-substituted macrocyclic hexaoxazole (6OTD) 2, and its possible interaction with telomeric G4. 


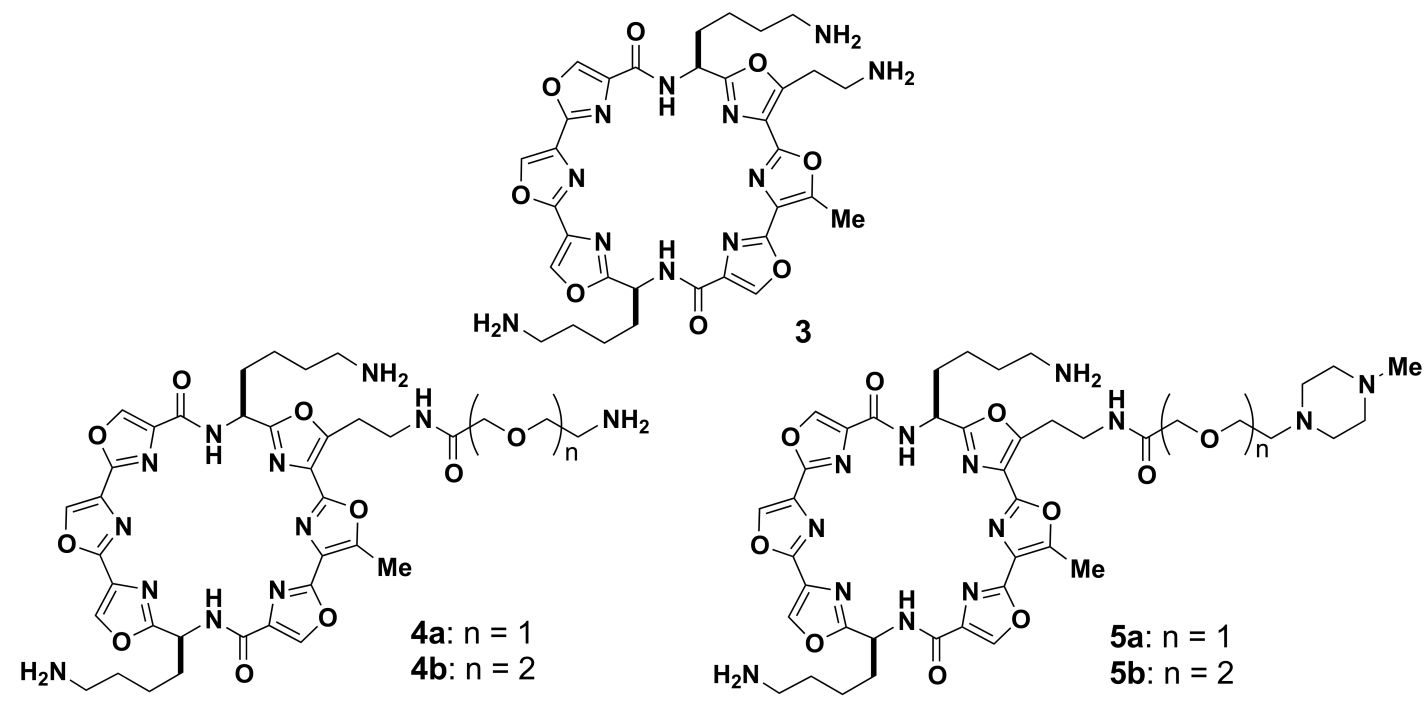

Figure 2. Structures of novel 6OTD analogs 3-5 targeting the G-quartet and the groove of telomeric G4.

\subsection{Synthesis of Tri-Substituted 6OTD Derivatives 3-5}

We synthesized tri-substituted 6OTD derivatives 3-5 as summarized in Schemes 1 and 2. Firstly, the synthesis of tri-amine 3 was carried out. Briefly, the bis-Boc-protected tri-substituted 6OTD 14 was synthesized from trioxazoles $\mathbf{6}$ and $\mathbf{8}$ based upon the procedure developed by our group [41,42]. Then, the two Boc groups in $\mathbf{1 4}$ were deprotected with TFA to give tri-amine $\mathbf{3}$ in $81 \%$ yield from $\mathbf{1 3}$. Compounds 4 and 5 were synthesized as follows: The amine 14 was reacted with carboxylic acids 15a $(n=1)$ and $\mathbf{1 5 b}(n=2)$ bearing an azide functional group in the presence of EDCI and HOBt, and the corresponding amides $\mathbf{1 6 a}$ and $\mathbf{1 6} \mathbf{b}$ were obtained in $42 \%$ and $39 \%$ yields, respectively. After reduction of the azide group in $\mathbf{1 6}$ under hydrogen in the presence of $\mathrm{Pd} / \mathrm{C}$ as a catalyst, the resulting amine $\mathbf{1 7}$ was deprotected with TFA to give tri-amines $4 \mathbf{a}$ and $\mathbf{4 b}$ in $94 \%$ and $90 \%$ yields from $\mathbf{1 6}$, respectively. The tri-substituted L2H2-6OTD derivatives of $5 \mathbf{a}$ and $5 \mathbf{b}$, bearing an $N$-methylpiperazine-substituted side chain, were synthesized similarly to 4 from amine 14 and carboxylic acids 18a $(n=1)$ and $\mathbf{1 8 b}(n=2)$. 


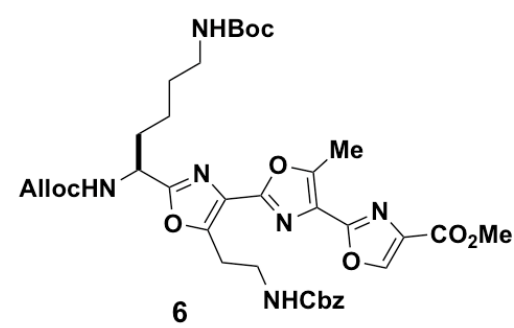

6

$\downarrow \mathrm{LiOH} \cdot \mathrm{H}_{2} \mathrm{O}$

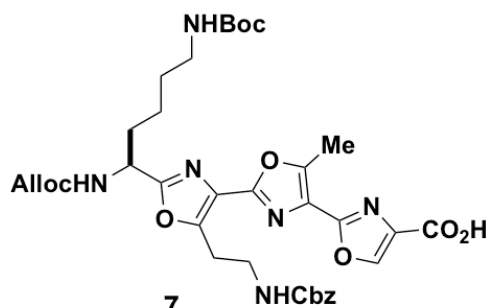

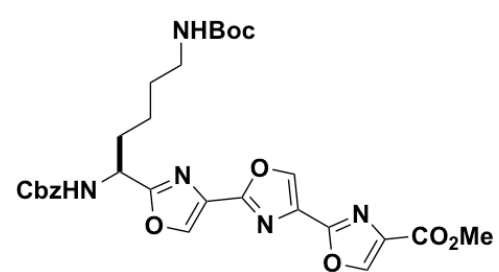

8

$\mathrm{Pd} / \mathrm{C}, \mathrm{H}_{2}$

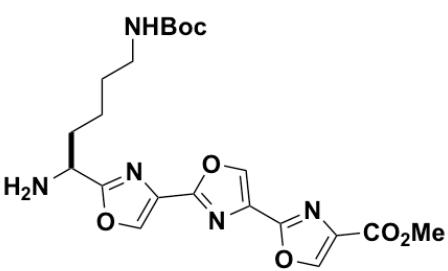

9

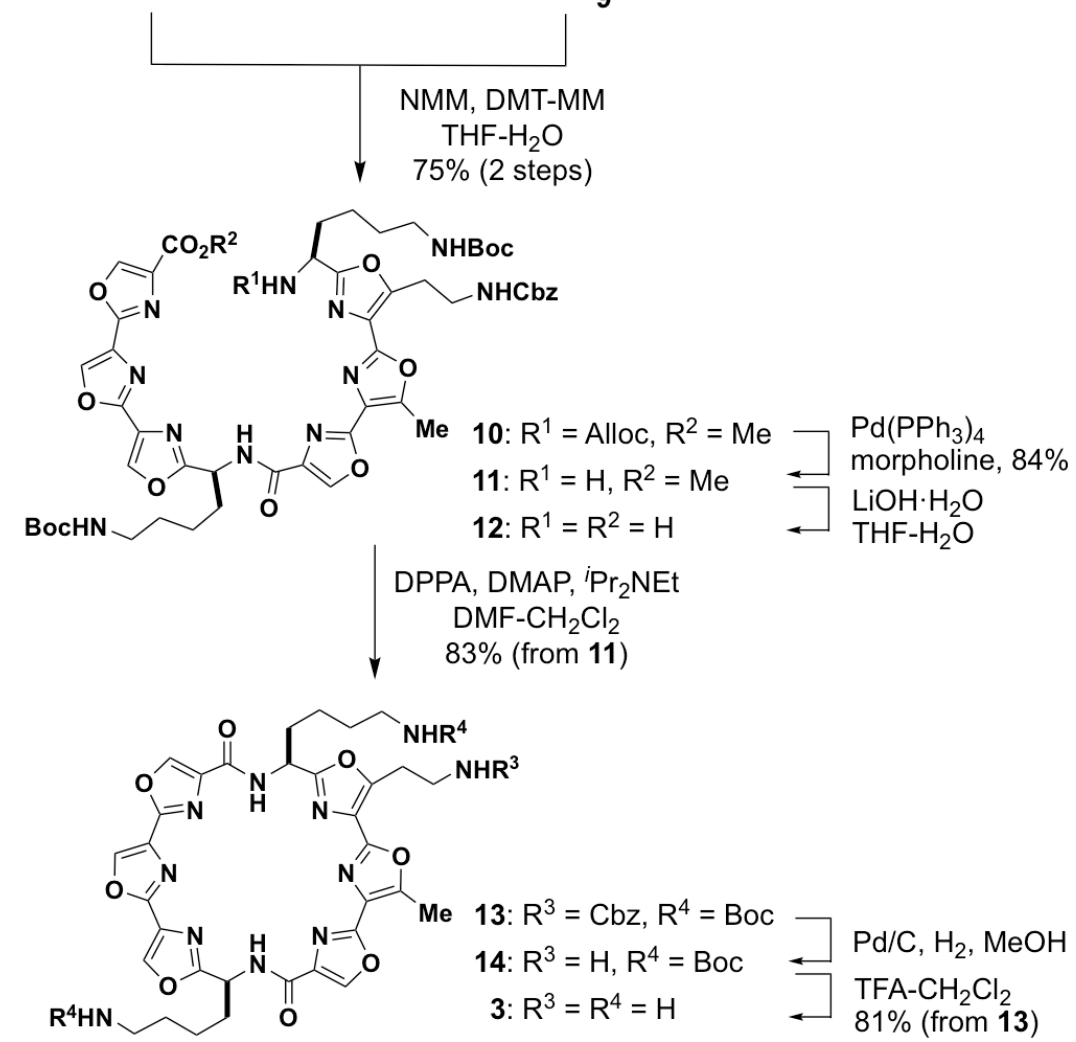

Scheme 1. Synthesis of tri-aminoalkyl-substituted 6OTD 3. 

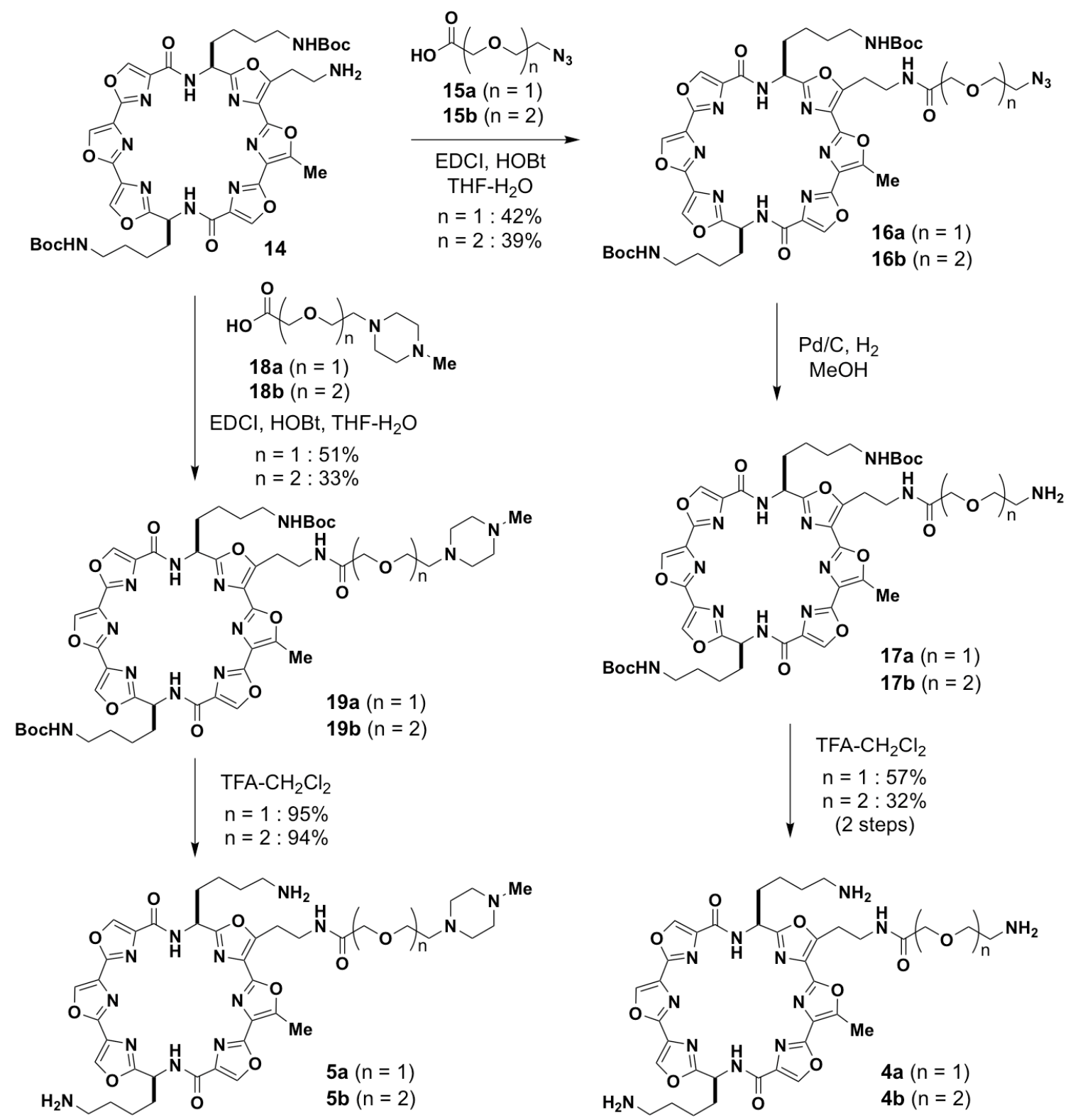

Scheme 2. Synthesis of tri-substituted 6OTDs 4 and 5.

2.3. Stabilization Abilities of 3-5 for G4-Forming DNA Sequences by Fluorescence Resonance Energy Transfer (FRET) Melting Analysis

Next, the abilities of 3-5 to stabilize telomere, c-kit, and K-ras G4-forming DNA sequences were compared with that of L2H2-6M(2)OTD 1 by means of FRET melting assay, with results shown in Table 1 [43]. In the case of telomeric $\mathrm{G} 4$, telo21, the $\Delta T_{\mathrm{m}}$ value was increased by ligands $\mathbf{3}-\mathbf{5}$ bearing additional side chains compared with L2H2-6M(2)OTD 1, except for with 5a. In regards to other G4-forming DNA sequences, slight increases of $\Delta T_{\mathrm{m}}$ for $c$-kit were observed with ligands 3, 4a, and $4 \mathbf{b}$, while significant decreases were observed with $5 \mathbf{a}$ and $5 \mathbf{b}$. In the case of $K$-ras, the $\Delta T_{\mathrm{m}}$ values were slightly decreased with ligands $\mathbf{5 a}$ and $\mathbf{5 b}$, respectively. These results suggest that $\mathbf{5 b}$ interacts selectively with telomeric G4 over the G4-forming DNA sequences of $c-k i t$ and $K$-ras, among the ligands and DNA sequences we examined. 
Table 1. Fluorescence resonance energy transfer (FRET) melting analysis of 6OTDs 3-5 (1.0 $\mu \mathrm{M})$ with G4-forming DNA sequences $(0.2 \mu \mathrm{M})$ *.

\begin{tabular}{ccccccc}
\hline \multirow{2}{*}{ DNA } & \multicolumn{7}{c}{$\Delta \mathbf{T}_{\mathbf{m}}$ Values $\left({ }^{\circ} \mathbf{C}\right)$} \\
\cline { 2 - 7 } & L2H2-6M(2)OTD (1) & $\mathbf{3}$ & $\mathbf{4 a}$ & $\mathbf{4 b}$ & $\mathbf{5 a}$ & $\mathbf{5 b}$ \\
\hline telo21 & $18.1 \pm 0.0$ & $23.4 \pm 0.1$ & $23.5 \pm 0.2$ & $24.4 \pm 0.5$ & $18.0 \pm 0.9$ & $26.2 \pm 0.3$ \\
c-kit & $18.3 \pm 0.4$ & $20.8 \pm 0.2$ & $19.5 \pm 0.3$ & $20.7 \pm 0.1$ & $12.9 \pm 0.6$ & $12.1 \pm 0.3$ \\
K-ras & $10.3 \pm 0.4$ & $13.0 \pm 1.0$ & $11.9 \pm 0.2$ & $13.5 \pm 0.2$ & $9.0 \pm 0.2$ & $8.2 \pm 0.4$ \\
dsDNA & $0.1 \pm 0.0$ & $0.0 \pm 0.1$ & $0.1 \pm 0.0$ & $0.0 \pm 0.2$ & $0.0 \pm 0.1$ & $0.0 \pm 0.1$ \\
\hline \multicolumn{7}{c}{${ }^{*}$ Melting temperatures were calculated from the average and SEM of at least three experiments. }
\end{tabular}

\subsection{Docking Studies of Ligands $\mathbf{3}, \mathbf{4}$, and $\mathbf{5 b}$ with Telomeric G4}

To gain insight into the differences in the stabilizing abilities of $\mathbf{3}, \mathbf{4}$, and $\mathbf{5 b}$, docking studies with telomeric G4 were carried out. Since the three topologies of telomeric DNA [44] (i.e., anti-parallel, hybrid, and parallel) are affected by ionic conditions, solvents, and ligands, we first analyzed the telomeric G4 topologies induced by ligands $\mathbf{3}-\mathbf{5}$ by means of Circular Dichroism (CD) spectroscopy [45]. As shown in Figure 3, telomeric G4 was induced predominantly into the parallel form in the presence of ligands 3,4 , and $\mathbf{5 b}$ under cation-free conditions. On the other hand, in case of ligand $\mathbf{5 a}$, the telomeric G4 was induced mainly into parallel-type topology, but did not show a single, distinctive spectrum, in contrast to the other ligands [46].

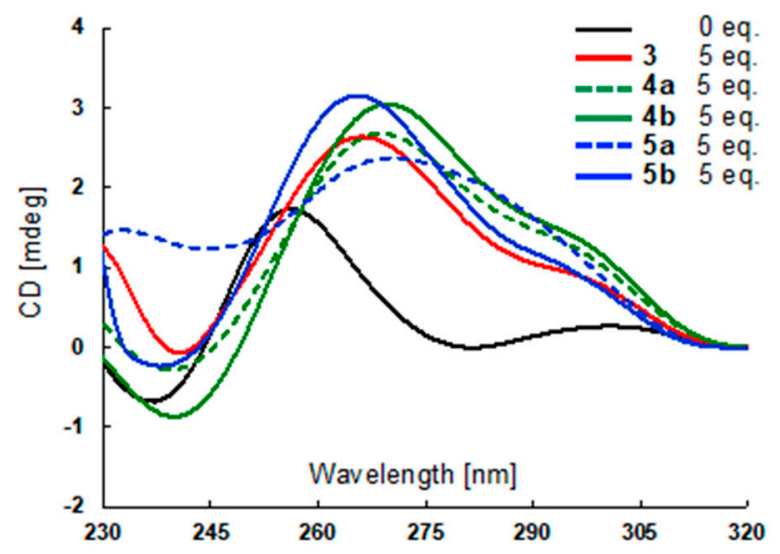

Figure 3. Circular Dichroism (CD) spectra of 3, 4 and 5 in the presence of telomeric G4.

With the $\mathrm{CD}$ analysis results in hand, docking studies were carried out between parallel-type telomeric G4 and ligands 3, 4, and 5b. The docking energies between the ligands and parallel-type telomeric G4 were calculated, and the distribution of the energies for the top 100 poses is shown in Figure 4 [47]. In these calculations, $5 \mathbf{b}$ mainly showed the lowest-energy docking (ca. -9 to $-10.5 \mathrm{kcal} / \mathrm{mol}$ ) among the ligands tested. The next lowest energy was found for $\mathbf{4} \mathbf{b}$, followed by $\mathbf{4 a}$, and 3, which is consistent with the results of the FRET melting analysis, which were shown in Table 1. 


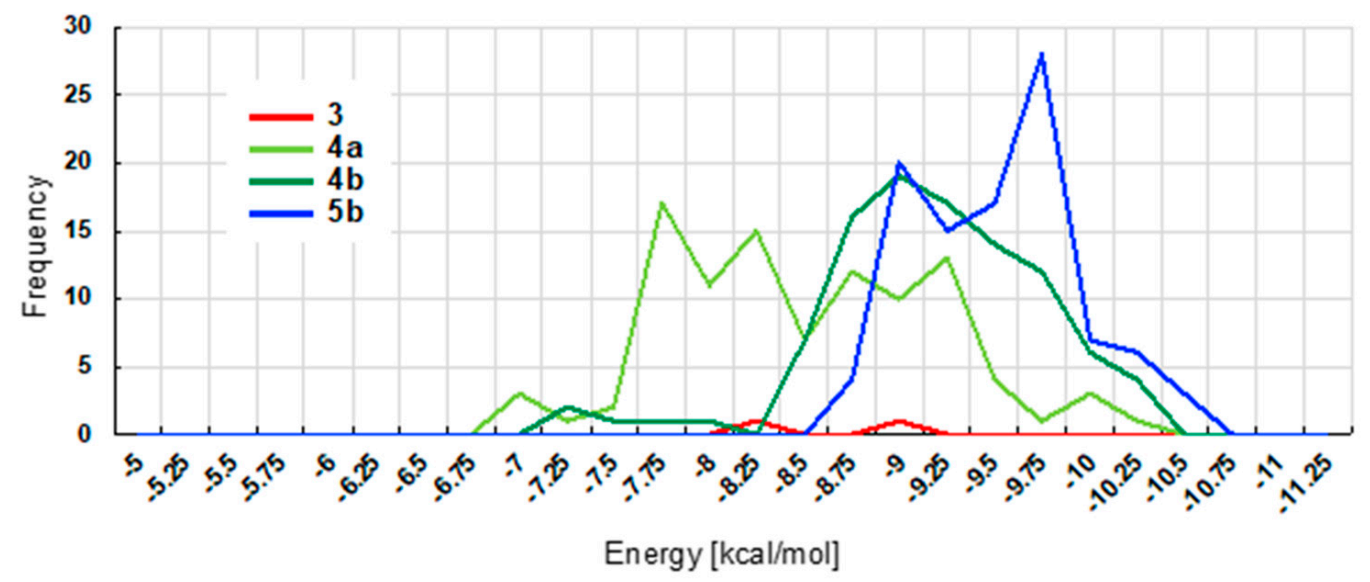

Figure 4. Distribution of docking energies of the top 100 poses of $\mathbf{3}, \mathbf{4 a}, \mathbf{4 b}$, and $\mathbf{5 b}$ with parallel-type telomeric G4.

Docking models with the lowest energy scores for ligands $\mathbf{3}, \mathbf{4}$, and $\mathbf{5 b}$ are depicted in Figure 5. In every case, the hexaoxazole moiety in the ligands stacks with the G-quartet, and the newly introduced side chain is suggested to interact with the groove in the parallel-type telomeric G4. The two nitrogens of piperazine in the side chain in $\mathbf{5 b}$ were suggested to interact efficiently with the phosphate backbone of DNA, which may contribute to $5 \mathbf{b}$ 's potent stabilizing ability for telomeric DNA, compared to $\mathbf{3}$ and $\mathbf{4}$. In the case of $\mathbf{5 a}$, the newly introduced side chain may have an inappropriate chain length for binding with the groove, resulting in a weak stabilizing ability for parallel-type telomeric G4.

a) Telomeric $\mathrm{G} 4$ with 3

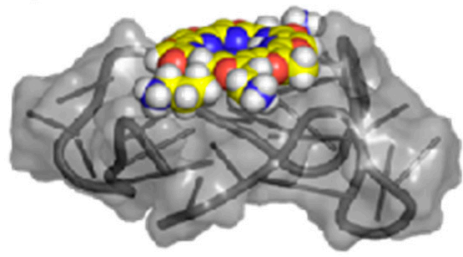

c) Telomeric G4 with $\mathbf{4 b}$

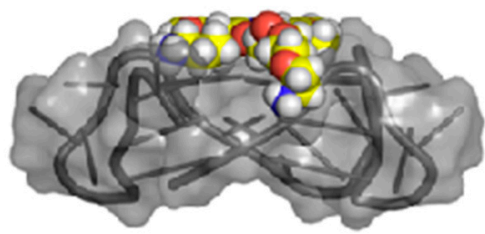

b) Telomeric $\mathbf{G} 4$ with $\mathbf{4 a}$

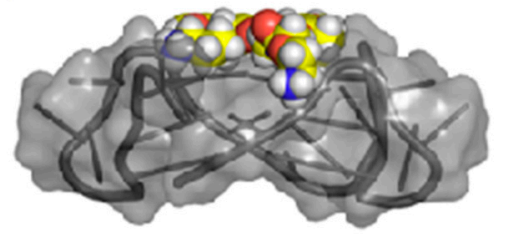

d) Telomeric G4 with $\mathbf{5 b}$

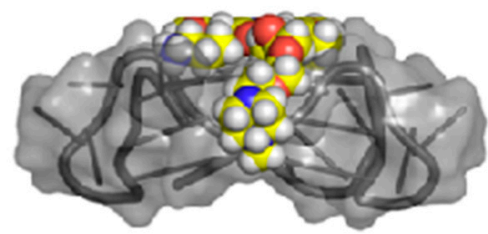

Figure 5. Docking models of parallel-type telomeric G4 with compounds (a) 3, (b,c) 4, and (d) 5 b.

\section{Materials and Methods}

\subsection{General}

Flash chromatography was performed on Silica gel 60 (spherical, particle size $0.040-0.100 \mathrm{~mm}$; Kanto Co., Inc., Tokyo, Japan). Preparative-TLC (PLC) was performed using PLC Silica gel 60 F254 (0.5 mm, Merck Ltd., Darmstadt, Germany). Optical rotations were measured on a JASCO P-2200 polarimeter. ${ }^{1} \mathrm{H}$ - and ${ }^{13} \mathrm{C}-\mathrm{NMR}$ spectra were recorded on JEOL JNM-AL300 (300 MHz), JEOL JNM-ECX $400(400 \mathrm{MHz})$, and JEOL JNM-ECA $500(500 \mathrm{MHz})$. The spectra were referenced internally according to the residual solvent signals of $\mathrm{CDCl}_{3}\left({ }^{1} \mathrm{H}-\mathrm{NMR}, \delta=7.26 \mathrm{ppm} ;{ }^{13} \mathrm{C}-\mathrm{NMR}, \delta=77.0 \mathrm{ppm}\right)$ and DMSO- $d_{6}\left({ }^{1} \mathrm{H}-\mathrm{NMR}, \delta=2.50 \mathrm{ppm} ;{ }^{13} \mathrm{C}-\mathrm{NMR}, \delta=39.5 \mathrm{ppm}\right)$. Dates for ${ }^{1} \mathrm{H}-\mathrm{NMR}$ were recorded as follows: Chemical shift $(\delta, \mathrm{ppm})$, multiplicity (s, singlet; $\mathrm{t}$, triplet; $\mathrm{m}$, multiplet; br, broad), integration, 
and coupling constant $(\mathrm{Hz})$. Dates for ${ }^{13} \mathrm{C}-\mathrm{NMR}$ were reported in terms of chemical shift $(\delta, \mathrm{ppm})$. Mass spectra were recorded on a JEOL JMS-T100LC spectrometer with ESI-MS mode, using MeOH as solvent.

\subsection{Synthesis Methods}

Compound 10: To a solution of trioxazole 6 [41] $(1.00 \mathrm{~g}, 1.36 \mathrm{mmol})$ in THF- $\mathrm{H}_{2} \mathrm{O}(3: 1,80 \mathrm{~mL})$ was added $\mathrm{LiOH} \cdot \mathrm{H}_{2} \mathrm{O}(85.5 \mathrm{mg}, 2.04 \mathrm{mmol})$ at room temperature, and stirred for $30 \mathrm{~min}$. The reaction was acidified with $3 \mathrm{~N} \mathrm{HCl}$ to give carboxylic acid 7 as a THF- $\mathrm{H}_{2} \mathrm{O}$ solution, which was used without further purification. To a solution of trioxazole 8 [42] $(1.20 \mathrm{~g}, 1.40 \mathrm{mmol})$ in $\mathrm{MeOH}-\mathrm{THF}(1: 3,80 \mathrm{~mL})$ was added $10 \% \mathrm{Pd} / \mathrm{C}(120 \mathrm{mg})$, and the mixture was stirred at room temperature under a hydrogen atmosphere for $10 \mathrm{~min}$. The reaction mixture was filtered through a pad of Celite ${ }^{\circledR}$ (FUJIFILM Wako Pure Chemical Co., Osaka, Japan) and eluted with $\mathrm{CHCl}_{3}-\mathrm{MeOH}(9: 1)$. The filtrates were concentrated in vacuo to give an amine 9 , which was used without further purification. To a solution of carboxylic acid 7 (THF- $\left.\mathrm{H}_{2} \mathrm{O}\right)$ was added NMM $(451 \mu \mathrm{L}, 4.08 \mathrm{mmol})$, DMT-MM (1.12 g, $\left.4.08 \mathrm{mmol}\right)$, and amine 9, and the mixture was stirred at room temperature. After being stirred overnight, to the reaction mixture was added $\mathrm{H}_{2} \mathrm{O}$, and the organic layer was extracted with $\mathrm{CHCl}_{3}$, washed with $1.2 \mathrm{~N} \mathrm{HCl}$, dried over $\mathrm{MgSO}_{4}$, and filtered. The filtrates were concentrated in vacuo, and the residue was purified by column chromatography on silica gel $\left(\mathrm{CHCl}_{3}: \mathrm{MeOH}=200: 1\right)$ to give bistrioxazole $10(1.20 \mathrm{~g}, 75 \%$, two steps). Spectral data for 10: $[\alpha]_{D}^{25}=-17.8\left(c\right.$ 1.1, $\left.\mathrm{CHCl}_{3}\right) ;{ }^{1} \mathrm{H}-\mathrm{NMR}\left(400 \mathrm{MHz}, \mathrm{CDCl}_{3}\right) \delta 8.31-8.27(\mathrm{~m}, 4 \mathrm{H})$, $8.06(\mathrm{~d}, J=8.7 \mathrm{~Hz}, 1 \mathrm{H}), 7.26-7.21(\mathrm{~m}, 5 \mathrm{H}), 5.90(\mathrm{~m}, 1 \mathrm{H}), 5.47(\mathrm{~m}, 1 \mathrm{H}), 5.33-5.20(\mathrm{~m}, 2 \mathrm{H}), 5.03-4.94(\mathrm{~m}$, $3 \mathrm{H}), 4.66-4.57(\mathrm{~m}, 3 \mathrm{H}), 3.95(\mathrm{~s}, 3 \mathrm{H}), 3.73-3.62(\mathrm{~m}, 2 \mathrm{H}), 3.45(\mathrm{t}, J=6.4 \mathrm{~Hz}, 2 \mathrm{H}), 3.10(\mathrm{br}, 4 \mathrm{H}), 2.71(\mathrm{~s}$, 3H), 2.26-1.78 (m, 4H), 1.52-1.40 (m, 26H); ${ }^{13} \mathrm{C}-\mathrm{NMR}\left(100 \mathrm{MHz}, \mathrm{CDCl}_{3}\right) \delta 165.0,161.1,160.2,156.3$, $155.9,155.8,155.2,151.2,150.9,143.8,141.1,139.5,139.2,139.1,136.4,136.1,134.1,132.4,130.5,129.5$, $128.2,127.8,127.6,126.5,124.3,117.7,78.9,78.8,77.2,66.5,65.8,54.5,52.2,49.0,46.7,40.0,39.8,38.7$, 33.4, 32.5, 29.3, 28.2, 26.7, 22.7, 22.3, 11.6; HRMS (ESI, M + Na) calcd. for $\mathrm{C}_{56} \mathrm{H}_{67} \mathrm{~N}_{11} \mathrm{O}_{17} \mathrm{Na} 1188.4614$, found 1188.4628 .

Compound 11: To a solution of bistrioxazole 10 (400 mg, $340 \mu \mathrm{mol})$ in THF (100 mL) was added morpholine $(298 \mu \mathrm{L}, 3.43 \mathrm{mmol})$ and $\mathrm{Pd}\left(\mathrm{PPh}_{3}\right)_{4}(118 \mathrm{mg}, 100 \mu \mathrm{mol})$ at room temperature under argon atmosphere and stirred for $10 \mathrm{~min}$. The reaction mixture was concentrated in vacuo, and the residue was purified by column chromatography on silica gel $\left(\mathrm{CHCl}_{3}: \mathrm{MeOH}=100: 3\right)$ to give amine $11(350 \mathrm{mg}$, 84\%). Spectral data for 11: $[\alpha]_{D}^{25}=+5.33\left(\right.$ c 1.4, $\left.\mathrm{CHCl}_{3}\right) ;{ }^{1} \mathrm{H}-\mathrm{NMR}\left(400 \mathrm{MHz}, \mathrm{CDCl}_{3}\right) \delta 8.31(\mathrm{~s}, 1 \mathrm{H}), 8.30$ $(\mathrm{s}, 1 \mathrm{H}), 8.27(\mathrm{~s}, 1 \mathrm{H}), 8.07(\mathrm{~d}, J=8.7 \mathrm{~Hz}, 1 \mathrm{H}), 7.26-7.22(\mathrm{~m}, 7 \mathrm{H}), 5.50-5.44(\mathrm{~m}, 1 \mathrm{H}), 5.24(\mathrm{br}, 1 \mathrm{H}), 5.04-4.96$ $(\mathrm{m}, 2 \mathrm{H}), 4.66(\mathrm{br}, 1 \mathrm{H}), 4.55(\mathrm{br}, 1 \mathrm{H}), 4.04(\mathrm{t}, J=6.9 \mathrm{~Hz}, 2 \mathrm{H}), 3.95(\mathrm{~s}, 3 \mathrm{H}), 3.75-3.44(\mathrm{~m}, 4 \mathrm{H}), 3.12-3.10(\mathrm{br}$, 4H), 2.72 (s, 3H), 2.25-0.86 (m, 30H); ${ }^{13} \mathrm{C}-\mathrm{NMR}\left(100 \mathrm{MHz}, \mathrm{CDCl}_{3}\right) \delta 166.3,165.1,161.2,160.3,156.3$, 155.9, 155.3, 151.1, 143.8, 141.1, 139.6, 139.2, 136.5, 136.1, 134.3, 130.6, 129.6, 128.3, 128.0, 127.7, 79.0, 78.9, 77.2, 70.5, 66.7, 52.3, 49.9, 46.8, 40.2, 40.0, 38.9, 35.4, 32.6, 29.7, 29.3, 28.3, 26.8, 22.9, 22.7, 11.8; HRMS (ESI, $\mathrm{M}+\mathrm{Na}$ ) calcd. for $\mathrm{C}_{52} \mathrm{H}_{63} \mathrm{~N}_{11} \mathrm{O}_{15} \mathrm{Na} 1104.4403$, found 1104.4370.

Compound 3: To a solution of $13(70.0 \mathrm{mg}, 66.7 \mu \mathrm{mol})$ in $\mathrm{MeOH}(6 \mathrm{~mL})$ was added 10\% Pd/C (50.0 mg), and the mixture was stirred at room temperature under a hydrogen atmosphere for $10 \mathrm{~min}$. The reaction mixture was filtered through a pad of Celite ${ }^{\circledR}$ and eluted with $\mathrm{CHCl}_{3}-\mathrm{MeOH}$ (9:1). The filtrates were concentrated in vacuo to give amine 14, which was used without further purification. The amine 14 was dissolved in $\mathrm{CH}_{2} \mathrm{Cl}_{2}$-TFA $(10: 1,11 \mathrm{~mL})$, and the resulting solution was stirred at room temperature for $10 \mathrm{~min}$. The reaction mixture was concentrated in vacuo to give 3 (38.7 $\mathrm{mg}, 81 \%$, two steps). Spectral data for 3: $[\alpha]_{D}^{25}=+46.1\left(c\right.$ 1.1, MeOH); ${ }^{1} \mathrm{H}-\mathrm{NMR}\left(400 \mathrm{MHz}, \mathrm{DMSO}-d_{6}\right) \delta 9.14-9.12(\mathrm{~m}, 2 \mathrm{H}), 8.92-8.90$ $(\mathrm{m}, 2 \mathrm{H}), 8.33-8.27(\mathrm{~m}, 2 \mathrm{H}), 8.15(\mathrm{br}, 2 \mathrm{H}), 7.75(\mathrm{br}, 4 \mathrm{H}), 5.45(\mathrm{dt}, J=12.8,5.5 \mathrm{~Hz}, 1 \mathrm{H}), 5.37(\mathrm{dt}, J=12.8$, $5.5 \mathrm{~Hz}, 1 \mathrm{H}), 3.75-3.29(\mathrm{~m}, 4 \mathrm{H}), 2.81-2.73(\mathrm{~m}, 7 \mathrm{H}), 2.11-1.88(\mathrm{~m}, 4 \mathrm{H}), 1.54-1.19(\mathrm{~m}, 8 \mathrm{H}){ }^{13} \mathrm{C}-\mathrm{NMR}(100$ MHz, DMSO-d $)_{6} \delta 164.4,162.0,158.9,158.8,158.7,158.3,155.7,155.3,155.1,154.6,151.9,149.8,142.5$, 142.4, 141.8, 141.1, 136.0, 136.0, 129.7, 128.5, 125.9, 123.8, 118.4, 47.2, 47.1, 36.9, 33.3, 26.7, 23.9, 20.9, 11.5; HRMS (ESI, $\mathrm{M}+\mathrm{H}$ ) calcd. for $\mathrm{C}_{33} \mathrm{H}_{38} \mathrm{~N}_{11} \mathrm{O}_{8} 716.2904$, found 716.2918. 
Compound 16a: To a solution of 15a $(10.0 \mathrm{mg}, 66.6 \mu \mathrm{mol})$ in $\mathrm{THF}-\mathrm{H}_{2} \mathrm{O}(2: 1,3 \mathrm{~mL})$ was added EDCI $(38.0 \mathrm{mg}, 200 \mu \mathrm{mol})$ and $\mathrm{HOBt}(27.0 \mathrm{mg}, 200 \mu \mathrm{mol})$ at room temperature. After stirring for $1 \mathrm{~h}$, to the reaction mixture was added amine $14(61.0 \mathrm{mg}, 66.6 \mu \mathrm{mol})$. The mixture was stirred for $24 \mathrm{~h}$ and quenched with $1.2 \mathrm{~N} \mathrm{HCl}$, and the organic layer was extracted with $\mathrm{CHCl}_{3}$, dried over $\mathrm{MgSO}_{4}$, and filtered. The filtrates were concentrated in vacuo, and the residue was purified by column chromatography on silica gel $\left(\mathrm{CHCl}_{3}: \mathrm{MeOH}=80: 1\right)$ to give $16 \mathrm{a}(29.0 \mathrm{mg}, 42 \%)$. Spectral data for 16a: $[\alpha]_{D}^{25}=-14.5\left(c\right.$ 0.58, $\left.\mathrm{CHCl}_{3}\right) ;{ }^{1} \mathrm{H}-\mathrm{NMR}\left(300 \mathrm{MHz}, \mathrm{DMSO}-d_{6}\right) \delta 9.12(\mathrm{~s}, 1 \mathrm{H}), 9.09(\mathrm{~s}, 1 \mathrm{H}), 8.91(\mathrm{~s}, 1 \mathrm{H})$, $8.89(\mathrm{~s}, 1 \mathrm{H}), 8.34-8.27(\mathrm{~m}, 2 \mathrm{H}), 7.98(\mathrm{t}, J=6.2 \mathrm{~Hz}, 1 \mathrm{H}), 6.77(\mathrm{t}, J=5.2 \mathrm{~Hz}, 2 \mathrm{H}), 5.42(\mathrm{dt}, J=12.4,7.2$ $\mathrm{Hz}, 1 \mathrm{H}), 5.32(\mathrm{dt}, J=12.4,7.2 \mathrm{~Hz}, 1 \mathrm{H}), 3.84(\mathrm{~d}, J=15.5 \mathrm{~Hz}, 1 \mathrm{H}), 3.78(\mathrm{~d}, J=15.1 \mathrm{~Hz}, 1 \mathrm{H}), 3.61-3.27$ (m, 6H), 2.84 (br, 4H), 2.75 (s, 3H), 2.04 (br, 2H), 1.90 (br, 2H), 1.33-1.03 (m, 28H); ${ }^{13} \mathrm{C}-\mathrm{NMR}(125 \mathrm{MHz}$, DMSO-d $\left.{ }_{6}\right) \delta 169.0,164.5,162.5,158.9,158.7,155.7,155.5,154.5,154.3,152.5,151.0,142.5,142.3,141.7$, 141.0, 136.0, 135.9, 129.8, 128.4, 124.8, 124.6, 79.2, 77.2, 69.7, 69.5, 49.8, 47.4, 47.2, 36.6, 33.5, 33.4, 29.2, 28.2, 26.1, 21.1, 11.5; HRMS (ESI, $\mathrm{M}+\mathrm{Na}$ ) calcd. for $\mathrm{C}_{47} \mathrm{H}_{58} \mathrm{~N}_{14} \mathrm{O}_{14} \mathrm{Na}$ 1065.4155, found 1065.4143.

Compound 4a: To a solution of $16 a(20.0 \mathrm{mg}, 19.2 \mu \mathrm{mol})$ in $\mathrm{MeOH}(4 \mathrm{~mL})$ was added $10 \% \mathrm{Pd} / \mathrm{C}$ $(20.0 \mathrm{mg})$, and the mixture was stirred at room temperature under a hydrogen atmosphere for $30 \mathrm{~min}$. The reaction mixture was filtered through a pad of Celite ${ }^{\circledR}$ and eluted with $\mathrm{CHCl}_{3}-\mathrm{MeOH}(9: 1)$. The filtrates were concentrated in vacuo to give amine 17a, which was used without further purification. The amine 17a was dissolved in $\mathrm{CH}_{2} \mathrm{Cl}_{2}$-TFA $(2: 1,3 \mathrm{~mL})$, and the resulting solution was stirred at room temperature for $10 \mathrm{~min}$. The reaction mixture was concentrated in vacuo to give $4 \mathbf{a}(8.90 \mathrm{mg}$, $57 \%$, two steps). Spectral data for $4 \mathbf{a}:[\alpha]_{D}^{25}=+87.8(c 2.3, \mathrm{MeOH}) ;{ }^{1} \mathrm{H}-\mathrm{NMR}\left(500 \mathrm{MHz}, \mathrm{DMSO}-d_{6}\right) \delta$ $9.15(\mathrm{~s}, 1 \mathrm{H}), 9.14(\mathrm{~s}, 1 \mathrm{H}), 8.94(\mathrm{~s}, 1 \mathrm{H}), 8.92(\mathrm{~s}, 1 \mathrm{H}), 8.31(\mathrm{~d}, J=7.5 \mathrm{~Hz}, 1 \mathrm{H}), 8.28(\mathrm{~d}, J=7.5 \mathrm{~Hz}, 1 \mathrm{H}), 8.23(\mathrm{t}$, $J=5.7 \mathrm{~Hz}, 1 \mathrm{H}), 7.79(\mathrm{br}, 6 \mathrm{H}), 5.44(\mathrm{dt}, J=12.6,7.5 \mathrm{~Hz}, 1 \mathrm{H}), 5.34(\mathrm{dt}, J=12.6,7.5 \mathrm{~Hz}, 1 \mathrm{H}), 3.84(\mathrm{~d}, J=14.9$ $\mathrm{Hz}, 1 \mathrm{H}), 3.80(\mathrm{~d}, J=15.5 \mathrm{~Hz}, 1 \mathrm{H}), 3.62-3.21(\mathrm{~m}, 4 \mathrm{H}), 3.00-2.92(\mathrm{~m}, 2 \mathrm{H}), 2.77-2.73(\mathrm{~m}, 7 \mathrm{H}), 2.09-1.91(\mathrm{~m}$, 4H), 1.53-1.21 (m, 10H); ${ }^{13} \mathrm{C}-\mathrm{NMR}\left(125 \mathrm{MHz}, \mathrm{DMSO}-d_{6}\right) \delta 169.0,164.5,158.9,158.8,155.7,155.6,155.7$, 155.6, 154.6, 154.3, 152.5, 151.2, 142.6, 142.4, 141.9, 141.2, 136.0, 135.9, 129.7, 128.5, 124.7, 124.6, 69.7, 66.9, 47.2, 47.1, 38.6, 36.6, 33.5, 33.4, 26.7, 26.2, 20.9, 11.5; HRMS (ESI, M + Na) calcd. for $\mathrm{C}_{37} \mathrm{H}_{44} \mathrm{~N}_{12} \mathrm{O}_{10} \mathrm{Na}$ 839.3201, found 839.3196.

Compound 16b: To a solution of $\mathbf{1 5 b}(15.0 \mathrm{mg}, 77.3 \mu \mathrm{mol})$ in THF- $\mathrm{H}_{2} \mathrm{O}(4: 1,2.5 \mathrm{~mL})$ was added EDCI $(44.6 \mathrm{mg}, 230 \mu \mathrm{mol})$ and $\mathrm{HOBt}(31.3 \mathrm{mg}, 230 \mu \mathrm{mol})$ at room temperature. After stirring for $1 \mathrm{~h}$, to the reaction mixture was added amine $14(70.0 \mathrm{mg}, 77.3 \mu \mathrm{mol})$. The mixture was stirred for $24 \mathrm{~h}$ and quenched with $1.2 \mathrm{~N} \mathrm{HCl}$, and the organic layer was extracted with $\mathrm{CHCl}_{3}$, dried over $\mathrm{MgSO}_{4}$, and filtered. The filtrates were concentrated in vacuo, and the residue was purified by column chromatography on silica gel $\left(\mathrm{CHCl}_{3}: \mathrm{MeOH}=80: 1\right)$ to give $\mathbf{1 6 b}(32.7 \mathrm{mg}, 39 \%)$. Spectral data for $16 \mathbf{b}$ : $[\alpha]_{D}^{25}=-7.6\left(c\right.$ 1.7, $\left.\mathrm{CHCl}_{3}\right) ;{ }^{1} \mathrm{H}-\mathrm{NMR}\left(500 \mathrm{MHz}, \mathrm{DMSO}-d_{6}\right) \delta 9.12(\mathrm{~s}, 1 \mathrm{H}), 9.10(\mathrm{~s}, 1 \mathrm{H}), 8.91(\mathrm{~s}, 1 \mathrm{H}), 8.89$ $(\mathrm{s}, 1 \mathrm{H}), 8.34-8.28(\mathrm{~m}, 2 \mathrm{H}), 7.91(\mathrm{t}, J=6.3 \mathrm{~Hz}, 1 \mathrm{H}), 6.78(\mathrm{t}, J=5.7 \mathrm{~Hz}, 2 \mathrm{H}), 5.42(\mathrm{dt}, J=12.6,7.5 \mathrm{~Hz}, 1 \mathrm{H})$, $5.32(\mathrm{dt}, J=12.6,7.5 \mathrm{~Hz}, 1 \mathrm{H}), 3.80(\mathrm{~d}, J=15.5 \mathrm{~Hz}, 1 \mathrm{H}), 3.75(\mathrm{~d}, J=14.9 \mathrm{~Hz}, 1 \mathrm{H}), 3.61-3.22(\mathrm{~m}, 10 \mathrm{H})$, 2.90-2.80 (m, 4H), $2.74(\mathrm{~s}, 3 \mathrm{H}), 2.03(\mathrm{br}, 2 \mathrm{H}), 1.89$ (br, 2H), 1.41-0.85 (m, 28H); ${ }^{13} \mathrm{C}-\mathrm{NMR}(125 \mathrm{MHz}$, DMSO-d $\left.{ }_{6}\right) \delta 169.3,164.6,162.5,158.9,158.7,155.7,155.5,154.5,154.3,152.5,151.0,142.5,142.3,141.7$, 141.0, 136.0, 135.9, 129.8, 128.4, 124.8, 124.6, 79.2, 77.2, 70.1, 70.0, 69.3, 69.2, 50.0, 47.4, 47.2, 36.6, 33.5, 33.4, 29.2, 28.2, 29.1, 21.1, 21.0, 11.5; HRMS (ESI, $\mathrm{M}+\mathrm{Na}$ ) calcd. for $\mathrm{C}_{49} \mathrm{H}_{62} \mathrm{~N}_{14} \mathrm{O}_{15} \mathrm{Na} 1109.4417$, found 1109.4387 .

Compound $\mathbf{4 b}$ : To a solution of $\mathbf{1 6 b}(8.00 \mathrm{mg}, 7.35 \mu \mathrm{mol})$ in $\mathrm{MeOH}(1 \mathrm{~mL})$ was added $10 \% \mathrm{Pd} / \mathrm{C}$ $(10.0 \mathrm{mg})$, and the mixture was stirred at room temperature under a hydrogen atmosphere for $10 \mathrm{~min}$. The reaction mixture was filtered through a pad of Celite ${ }^{\circledR}$ and eluted with $\mathrm{CHCl}_{3}-\mathrm{MeOH}(9: 1)$. The filtrates were concentrated in vacuo to give amine $\mathbf{1 7 b}$, which was used without further purification. The amine $\mathbf{1 7} \mathbf{b}$ was dissolved in $\mathrm{CH}_{2} \mathrm{Cl}_{2}$-TFA $(2: 1,3 \mathrm{~mL})$, and the resulting solution was stirred at room temperature for $10 \mathrm{~min}$. The reaction mixture was concentrated in vacuo to give $4 \mathbf{b}(2.03 \mathrm{mg}$, $32 \%, 2$ steps). Spectral data for $4 \mathbf{b}:[\alpha]_{D}^{25}=+58.2($ c $2.1, \mathrm{MeOH}) ;{ }^{1} \mathrm{H}-\mathrm{NMR}\left(500 \mathrm{MHz}, \mathrm{DMSO}-d_{6}\right) \delta 9.15$ $(\mathrm{s}, 1 \mathrm{H}), 9.14(\mathrm{~s}, 1 \mathrm{H}), 8.94(\mathrm{~s}, 1 \mathrm{H}), 8.92(\mathrm{~s}, 1 \mathrm{H}), 8.30(\mathrm{~d}, J=7.5 \mathrm{~Hz}, 1 \mathrm{H}), 8.28(\mathrm{~d}, J=8.0 \mathrm{~Hz}, 1 \mathrm{H}), 8.07$ (br, 1H), $7.76(\mathrm{br}, 6 \mathrm{H}), 5.44(\mathrm{dt}, J=12.6,6.9 \mathrm{~Hz}, 1 \mathrm{H}), 5.35(\mathrm{dt}, J=12.6,7.5 \mathrm{~Hz}, 1 \mathrm{H}), 3.82-2.63(\mathrm{~m}, 19 \mathrm{H})$, 
2.09-1.87 (m, 4H), 1.53-1.22 (m, 10H); ${ }^{13} \mathrm{C}-\mathrm{NMR}\left(125 \mathrm{MHz}\right.$, DMSO-d $\left.d_{6}\right) \delta 169.5,164.5,162.4,159.0,158.8$, 158.4, 158.1, 155.7, 155.6, 154.6, 154.4, 152.6, 151.2, 142.6, 142.4, 141.9, 141.2, 137.4, 136.0, 135.9, 129.7, 128.9, 128.5, 128.2, 125.4, 124.7, 124.6, 69.9, 69.3, 66.7, 47.2, 47.0, 38.6, 36.6, 33.5, 33.4, 26.8, 26.1, 21.1, 21.0, 20.9, 11.6; HRMS (ESI, $\mathrm{M}+\mathrm{Na}$ ) calcd. for $\mathrm{C}_{39} \mathrm{H}_{48} \mathrm{~N}_{12} \mathrm{O}_{11} \mathrm{Na} 883.3463$, found 883.3479.

Compound 19a: To a solution of 18a $(9.60 \mathrm{mg}, 47.6 \mu \mathrm{mol})$ in $\mathrm{THF}^{-} \mathrm{H}_{2} \mathrm{O}(2: 1,3 \mathrm{~mL})$ was added EDCI $(27.4 \mathrm{mg}, 143 \mu \mathrm{mol})$ and $\mathrm{HOBt}(19.3 \mathrm{mg}, 143 \mu \mathrm{mol})$ at room temperature. After stirring for $1 \mathrm{~h}$, to the reaction mixture was added amine $14(43.6 \mathrm{mg}, 47.6 \mu \mathrm{mol})$. The mixture was stirred for $24 \mathrm{~h}$ and quenched with $1.2 \mathrm{~N} \mathrm{HCl}$, and the organic layer was extracted with $\mathrm{CHCl}_{3}$, dried over $\mathrm{MgSO}_{4}$, and filtered. The filtrates were concentrated in vacuo, and the residue was purified by column chromatography on silica gel $\left(\mathrm{CHCl}_{3}: \mathrm{MeOH}=10: 1\right)$ to give $19 \mathrm{a}(40.0 \mathrm{mg}, 76 \%)$. Spectral data for 19a: $[\alpha]_{D}^{25}=-0.25\left(c 1.0, \mathrm{CHCl}_{3}\right) ;{ }^{1} \mathrm{H}-\mathrm{NMR}\left(500 \mathrm{MHz}, \mathrm{DMSO}-d_{6}\right) \delta 9.12(\mathrm{~s}, 1 \mathrm{H}), 9.09(\mathrm{~s}, 1 \mathrm{H}), 8.91(\mathrm{~s}, 1 \mathrm{H}), 8.90$ $(\mathrm{s}, 1 \mathrm{H}), 8.32(\mathrm{~d}, J=8.0 \mathrm{~Hz}, 1 \mathrm{H}), 8.29(\mathrm{~d}, J=7.5 \mathrm{~Hz}, 1 \mathrm{H}), 8.12(\mathrm{t}, J=6.3 \mathrm{~Hz}, 1 \mathrm{H}), 6.78(\mathrm{t}, J=5.7 \mathrm{~Hz}, 2 \mathrm{H})$, $5.42(\mathrm{dt}, J=12.6,7.5 \mathrm{~Hz}, 1 \mathrm{H}), 5.31(\mathrm{dt}, J=12.6,7.5 \mathrm{~Hz}, 1 \mathrm{H}), 3.77(\mathrm{~d}, J=15.5 \mathrm{~Hz}, 1 \mathrm{H}), 3.72(\mathrm{~d}, J=14.9 \mathrm{~Hz}$, $1 \mathrm{H}), 3.62-3.24(\mathrm{~m}, 4 \mathrm{H}), 2.84(\mathrm{br}, 4 \mathrm{H}), 2.74(\mathrm{~s}, 3 \mathrm{H}), 2.36-1.88(\mathrm{~m}, 17 \mathrm{H}), 1.33-1.03(\mathrm{~m}, 28 \mathrm{H}) ;{ }^{13} \mathrm{C}-\mathrm{NMR}$ $\left(125 \mathrm{MHz}, \mathrm{DMSO}-d_{6}\right) \delta 169.7,164.5,162.5,158.9,158.7,155.7,155.5,154.5,154.3,152.5,151.0,142.5$, 142.3, 141.7, 141.0, 136.1, 136.0, 136.1, 135.9, 129.8, 128.5, 124.8, 124.7, 79.2, 77.3, 70.0, 68.5, 56.9, 54.7, 52.9, 47.4, 47.3, 45.7, 36.6, 33.4, 29.2, 28.2, 26.1, 21.1, 11.6; HRMS (ESI, $\mathrm{M}+\mathrm{Na}$ ) calcd. for $\mathrm{C}_{52} \mathrm{H}_{69} \mathrm{~N}_{13} \mathrm{O}_{14} \mathrm{Na}$ 1122.4985 , found 1122.4991 .

Compound 5a: To a solution of 19a $(6.70 \mathrm{mg}, 6.01 \mu \mathrm{mol})$ in $\mathrm{CH}_{2} \mathrm{Cl}_{2}(2 \mathrm{~mL})$ was added TFA $(1 \mathrm{~mL})$ at room temperature, and the mixture was stirred for $10 \mathrm{~min}$. The reaction mixture was concentrated in vacuo to give $5 \mathrm{a}(5.14 \mathrm{mg}, 95 \%)$. Spectral data for $5 \mathrm{a}:[\alpha]_{D}^{25}=+85.6\left(\right.$ c 3.4, MeOH) ${ }^{1} \mathrm{H}-\mathrm{NMR}(500 \mathrm{MHz}$, DMSO- $\left.d_{6}\right) \delta 9.16(\mathrm{~s}, 1 \mathrm{H}), 9.14(\mathrm{~s}, 1 \mathrm{H}), 8.94(\mathrm{~s}, 1 \mathrm{H}), 8.92(\mathrm{~s}, 1 \mathrm{H}), 8.32(\mathrm{~d}, J=7.5 \mathrm{~Hz}, 1 \mathrm{H}), 8.30(\mathrm{~d}, J=8.0 \mathrm{~Hz}$, $1 \mathrm{H}), 8.04(\mathrm{t}, J=6.3 \mathrm{~Hz}, 1 \mathrm{H}), 7.71(\mathrm{br}, 4 \mathrm{H}), 5.44(\mathrm{dt}, J=12.6,6.9 \mathrm{~Hz}, 1 \mathrm{H}), 5.34(\mathrm{dt}, J=12.6,7.5 \mathrm{~Hz}, 1 \mathrm{H}), 3.81$ $(\mathrm{d}, J=15.5 \mathrm{~Hz}, 1 \mathrm{H}), 3.76(\mathrm{~d}, J=14.9 \mathrm{~Hz}, 1 \mathrm{H}), 3.62-3.19(\mathrm{~m}, 15 \mathrm{H}), 2.98(\mathrm{br}, 2 \mathrm{H}), 2.75(\mathrm{br}, 7 \mathrm{H}), 2.07-1.87$ $(\mathrm{m}, 4 \mathrm{H}), 1.53-1.23(\mathrm{~m}, 10 \mathrm{H}){ }^{13} \mathrm{C}-\mathrm{NMR}\left(125 \mathrm{MHz}, \mathrm{DMSO}-d_{6}\right) \delta 169.1,164.5,162.5,159.0,158.8,158.5$, 155.7, 155.6, 154.6, 154.4, 152.6, 151.2, 142.6, 142.4, 141.9, 141.2, 137.4, 136.0, 135.9, 129.8, 129.0, 128.5, 128.3, 125.4, 124.8, 124.7, 117.7, 115.3, 69.5, 47.3, 47.1, 38.6, 33.5, 33.4, 26.8, 26.7, 26.0, 21.1, 21.0, 11.5; HRMS (ESI, $\mathrm{M}+\mathrm{H}$ ) calcd. for $\mathrm{C}_{42} \mathrm{H}_{54} \mathrm{~N}_{13} \mathrm{O}_{10} 900.4117$, found 900.4098 .

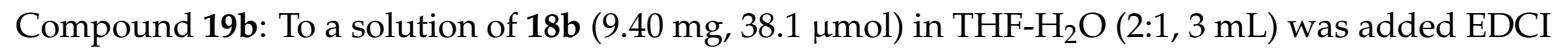
$(22.0 \mathrm{mg}, 114 \mu \mathrm{mol})$ and $\mathrm{HOBt}(15.0 \mathrm{mg}, 114 \mu \mathrm{mol})$ at room temperature. After stirring for $1 \mathrm{~h}$, to the reaction mixture was added amine $14(34.9 \mathrm{mg}, 38.1 \mu \mathrm{mol})$. The mixture was stirred for $24 \mathrm{~h}$ and quenched with $1.2 \mathrm{~N} \mathrm{HCl}$, and the organic layer was extracted with $\mathrm{CHCl}_{3}$, dried over $\mathrm{MgSO}_{4}$, and filtered. The filtrates were concentrated in vacuo, and the residue was purified by column chromatography on silica gel $\left(\mathrm{CHCl}_{3}: \mathrm{MeOH}=10: 1\right)$ to give $\mathbf{1 9 b}(14.4 \mathrm{mg}, 33 \%)$. Spectral data for $\mathbf{1 9 b}$ : $[\alpha]_{D}^{25}=-6.7\left(c 0.65, \mathrm{CHCl}_{3}\right) ;{ }^{1} \mathrm{H}-\mathrm{NMR}\left(500 \mathrm{MHz}, \mathrm{DMSO}-d_{6}\right) \delta 9.12(\mathrm{~s}, 1 \mathrm{H}), 9.09(\mathrm{~s}, 1 \mathrm{H}), 8.91(\mathrm{~s}, 1 \mathrm{H})$, $8.90(\mathrm{~s}, 1 \mathrm{H}), 8.34-8.28(\mathrm{~m}, 2 \mathrm{H}), 7.95(\mathrm{t}, J=5.7 \mathrm{~Hz}, 1 \mathrm{H}), 6.78(\mathrm{t}, J=5.7 \mathrm{~Hz}, 2 \mathrm{H}), 5.42(\mathrm{dt}, J=12.6,7.5 \mathrm{~Hz}$, $1 \mathrm{H}), 5.32(\mathrm{dt}, J=12.6,7.5 \mathrm{~Hz}, 1 \mathrm{H}), 3.79(\mathrm{~d}, J=15.5 \mathrm{~Hz}, 1 \mathrm{H}), 3.73(\mathrm{~d}, J=15.5 \mathrm{~Hz}, 1 \mathrm{H}), 3.60-3.16(\mathrm{~m}, 8 \mathrm{H})$, 2.86-2.84 (m, 4H), $2.74(\mathrm{~s}, 3 \mathrm{H}), 2.37-1.88(\mathrm{~m}, 17 \mathrm{H}), 1.33-1.04(\mathrm{~m}, 28 \mathrm{H}) ;{ }^{13} \mathrm{C}-\mathrm{NMR}\left(125 \mathrm{MHz}, \mathrm{DMSO}-d_{6}\right)$ $\delta 1169.9,165.1,163.0,162.8,159.4,159.2,156.2,156.0,155.0,154.8,153.0,151.5,143.0,142.8,142.2,141.5$, 136.6, 136.4, 130.3, 129.0, 125.3, 125.1, 79.7, 77.7, 70.5, 70.4, 69.9, 68.7, 57.7, 55.2, 53.5, 47.9, 47.7, 46.3, 37.1, 36.3, 34.0, 33.9, 29.7, 28.7, 26.6, 21.6, 21.5, 12.0; HRMS (ESI, $\mathrm{M}+\mathrm{Na}$ ) calcd. for $\mathrm{C}_{54} \mathrm{H}_{73} \mathrm{~N}_{13} \mathrm{O}_{15} \mathrm{Na}$ 1166.5247, found 1166.5238 .

Compound $5 \mathbf{b}$ : To a solution of $\mathbf{1 9 b}(14.4 \mathrm{mg}, 12.6 \mu \mathrm{mol})$ in $\mathrm{CH}_{2} \mathrm{Cl}_{2}(2 \mathrm{~mL})$ was added TFA $(1 \mathrm{~mL})$ at room temperature, and the mixture was stirred for $10 \mathrm{~min}$. The reaction mixture was concentrated in vacuo to give $5 \mathbf{b}(11.2 \mathrm{mg}, 94 \%)$. Spectral data for $5 \mathbf{b}:[\alpha]_{D}^{25}=+29.7(c 4.4, \mathrm{MeOH}) ;{ }^{1} \mathrm{H}-\mathrm{NMR}(500 \mathrm{MHz}$, DMSO- $\left.d_{6}\right) \delta 9.15(\mathrm{~s}, 1 \mathrm{H}), 9.14(\mathrm{~s}, 1 \mathrm{H}), 8.94(\mathrm{~s}, 1 \mathrm{H}), 8.92(\mathrm{~s}, 1 \mathrm{H}), 8.31(\mathrm{~d}, J=7.5 \mathrm{~Hz}, 1 \mathrm{H}), 8.28(\mathrm{~d}, J=7.5 \mathrm{~Hz}$, 1H), $8.02(\mathrm{br}, 1 \mathrm{H}), 7.73(\mathrm{br}, 4 \mathrm{H}), 5.45(\mathrm{dt}, J=12.6,7.5 \mathrm{~Hz}, 1 \mathrm{H}), 5.34(\mathrm{dt}, J=12.6,7.5 \mathrm{~Hz}, 1 \mathrm{H}), 3.80(\mathrm{~d}$, $J=15.5 \mathrm{~Hz}, 1 \mathrm{H}), 3.76(\mathrm{~d}, J=15.5 \mathrm{~Hz}, 1 \mathrm{H}), 3.60-3.19(\mathrm{~m}, 19 \mathrm{H}), 2.75(\mathrm{br}, 9 \mathrm{H}), 2.08-1.87(\mathrm{~m}, 4 \mathrm{H}), 1.53-1.17$ $(\mathrm{m}, 10 \mathrm{H}) ;{ }^{13} \mathrm{C}-\mathrm{NMR}\left(125 \mathrm{MHz}, \mathrm{DMSO}-d_{6}\right) \delta 169.4,164.5,162.4,158.8,155.7,152.7,151.2,141.9,141.2$, 
136.0, 128.9, 128.3, 124.8, 117.7, 115.3, 79.2, 69.9, 69.8, 69.3, 47.2, 47.1, 38.6, 33.5, 33.4, 26.8, 26.7, 26.1, 21.1, 21.0, 20.9, 11.5; HRMS (ESI, $\mathrm{M}+\mathrm{H}$ ) calcd. for $\mathrm{C}_{44} \mathrm{H}_{58} \mathrm{~N}_{13} \mathrm{O}_{11}$ 944.4379, found 944.4344 .

\subsection{FRET Melting Analysis}

Fluorescence resonance energy transfer (FRET) melting assay was performed with an excitation wavelength of $470-505 \mathrm{~nm}$ and a detection wavelength of 523-543 nm using the DNA Engine Option 2 Real-Time Cycler PCR detection system (Biorad, Hercules, CA, USA). The dual fluorescently labeled oligonucleotides were used in this protocol (Table S1). The donor fluorophore was 6-carboxyfluorescein (FAM) and the acceptor fluorophore was 6-carboxytetramethylrhodamine (TAMRA). All purified nucleotides (Sigma Genosys, Tokyo, Japan) were dissolved as stock solutions $(100 \mu \mathrm{M})$ in MilliQ water to be used without further purification. Further dilutions of the oligonucleotides were performed with a $60 \mathrm{mM}$ potassium cacodylate buffer ( $\mathrm{pH}$ 7.4), and FRET experiments were carried out with a $0.4 \mu \mathrm{M}$ oligonucleotide solution. Dual-labeled DNA was annealed by heating at $99{ }^{\circ} \mathrm{C}$ for $5 \mathrm{~min}$, and then slowly cooled to room temperature. Ligands were prepared as DMSO stock solutions $(10 \mathrm{mM})$ and diluted to $1 \mathrm{mM}$ using DMSO, and then diluted to $100 \mu \mathrm{M}$ using a $60 \mathrm{mM}$ potassium cacodylate buffer (pH 7.4). Next, the annealed DNA $(20 \mu \mathrm{L}, 0.4 \mu \mathrm{M})$ and the compound solution $(20 \mu \mathrm{L}$, $2 \mu \mathrm{M}$ ) were distributed across 96-well plates (Takara), with a total volume of $40 \mu \mathrm{L}$, with the labeled oligonucleotide $(0.2 \mu \mathrm{M})$ and the compound $(1.0 \mu \mathrm{M})$. The plates were incubated at $25{ }^{\circ} \mathrm{C}$ for $12 \mathrm{~h}$. Subsequent experiments used the following temperature procedure in RT-PCR, finishing as follows: $25{ }^{\circ} \mathrm{C}$ for $20 \mathrm{~min}$, and then a stepwise increase of $1{ }^{\circ} \mathrm{C}$ every minute from $25{ }^{\circ} \mathrm{C}$ until $99^{\circ} \mathrm{C}$. During the procedures, we measured the FAM fluorescence after each step. The change in the melting temperature at $1.0 \mu \mathrm{M}$ compound concentration $\left(\Delta T_{m}(1.0 \mu \mathrm{M})\right)$ was calculated from at least three experiments by subtraction of the blank from the averaged melting temperature of each compound.

\subsection{Spectrometry}

Circular Dichroism (CD) spectra were recorded on a J-720 spectropolarimeter (JASCO, Tokyo, Japan), using a quartz cell of $1 \mathrm{~mm}$ optical path length and an instrument scanning speed of $500 \mathrm{~nm} / \mathrm{min}$ with a response time of $1 \mathrm{~s}$, and over a wavelength range of $220-320 \mathrm{~nm}$. The oligonucleotide telo24 used in this protocol (Table S1) was dissolved as a $1.0 \mathrm{mM}$ stock solution in MilliQ water, to be used without further purification. Further dilution of the nucleotide was done with a $50 \mathrm{mM}$ Tris- $\mathrm{HCl}$ buffer (without ions or with $50 \mathrm{mM} \mathrm{KCl}$ ) from $1 \mathrm{mM}$ stock solutions, to give a concentration of $10 \mu \mathrm{M}$. The solution was annealed by heating at $99{ }^{\circ} \mathrm{C}$ for $5 \mathrm{~min}$, and then slowly cooled to room temperature, and then titrated into the oligonucleotide samples up to 5 mol equivalents using ligands, and incubated overnight. Finally, the CD spectra are representative of five averaged scans taken at $25^{\circ} \mathrm{C}$.

\subsection{Computational Analysis}

For the initial coordinates of compounds 3, 4, and 5, ionization and energy minimization were performed by the OPLS3 force field in the LigPrep Script in the Maestro (Schrödinger, LLC, New York, NY, USA). These minimized structures were employed as input structures for docking simulations. The human telomeric G4 structures with parallel-type topologies (PDB ID: 1KF1 and 3UYH) were refined for docking simulations using constrained energy refinements in the OPLS-AA force field (Schrödinger LLC). Docking simulations were performed using the Glide [48,49] SP docking program (Schrödinger, LLC, New York, NY, USA).

\section{Conclusions}

In conclusion, we have newly synthesized tri-substituted 6OTD derivatives $3-5$ by introducing aminoethyl, aminoethoxyethyl, and piperazinyethoxyethyl groups at C5 of L2H2-6M(2)OTD 1, respectively. Among these ligands, $\mathbf{5 b}$ stabilizes telomeric G4 preferentially over c-kit and K-ras G4s. Docking studies with parallel-type telomeric G4 revealed that the hexaoxazole moiety of $\mathbf{5 b}$ stacks with the G-quartet of telomeric G4, and the newly introduced side chain efficiently interacts with the groove. 
Supplementary Materials: The following are available online.

Author Contributions: Conceptualization, A.T.P. and K.N.; investigation, Y.M., K.I., and S.S.; molecular calculation, T.H. and B.H.; writing-original draft preparation, Y.M. and K.N.; writing-review and editing, K.N.

Funding: This research was funded by Grants-in-Aid for Scientific Research, Japan Society for the Promotion of Science (JSPS) (Scientific Research (B) 26282214 to K.N.; Scientific Research (C) 18K05349 to K.I.), a Grant-in-Aid for Scientific Research on Innovative Areas "Middle Molecular Strategy" (18H04387 to K.N.), a Grant-in-Aid for JSPS Research Fellow (16J08216 to Y.M.), funds from the Platform Project for Supporting Drug Discovery and Life Science Research (Basis for Supporting Innovative Drug Discovery and Life Science Research (BINDS)) from the Japan Agency for Medical Research and Development (AMED, JP18am0101114 to TH), and grants from Nanyang Technological University (to A.T.P.).

Conflicts of Interest: The authors declare no conflict of interest.

\section{References and Notes}

1. Davis, J.T. G-quartets 40 years later: From 5'-GMP to molecular biology and supramolecular chemistry. Angew. Chem. Int. Ed. 2004, 43, 668-698. [CrossRef] [PubMed]

2. Todd, A.K.; Johnston, M.; Neidle, S. Highly prevalent putative quadruplex sequence motifs in human DNA. Nucleic Acids Res. 2005, 33, 2901-2907. [CrossRef] [PubMed]

3. Huppert, J.L.; Balasubramanian, S. Prevalence of quadruplexes in the human genome. Nucleic Acids Res. 2005, 33, 2908-2916. [CrossRef] [PubMed]

4. Huppert, J.L.; Balasubramanian, S. G-quadruplexes in promoters throughout the human genome. Nucleic Acids Res. 2007, 35, 406-413. [CrossRef] [PubMed]

5. Huppert, J.L.; Bugaut, A.; Kumari, S.; Balasubramanian, S. G-quadruplexes: The beginning and end of UTRs. Nucleic Acids Res. 2008, 36, 6260-6268. [CrossRef] [PubMed]

6. Verma, A.; Yadav, V.K.; Basundra, R.; Kumar, A.; Chowdhury, S. Evidence of genome-wide G4 DNA-mediated gene expression in human cancer cells. Nucleic Acids Res. 2009, 37, 4194-4204. [CrossRef] [PubMed]

7. Chambers, V.S.; Marsico, G.; Boutell, J.M.; Di Antonio, M.; Smith, G.P.; Balasubramanian, S. High-throughput sequencing of DNA G-quadruplex structures in the human genome. Nat. Biotechnol. 2015, 33, 877-881. [CrossRef]

8. Paeschke, K.; Capra, J.A.; Zakian, V.A. DNA replication through G-quadruplex motifs is promoted by the Saccharomyces cerevisiae Pif1 DNA helicase. Cell 2011, 145, 678-691. [CrossRef]

9. Kanoh, Y.; Matsumoto, S.; Fukatsu, R.; Kakusho, N.; Kono, N.; Renard-Guillet, C.; Masuda, K.; Iida, K.; Nagasawa, K.; Shirahige, K.; et al. Rif1 binds to G quadruplexes and suppresses replication over long distances. Nat. Struct. Mol. Biol. 2015, 22, 889-897. [CrossRef]

10. Siddiqui-Jain, A.; Grand, C.L.; Bearss, D.J.; Hurley, L.H. Direct evidence for a G-quadruplex in a promoter region and its targeting with a small molecule to repress c-MYC transcription. Proc. Natl. Acad. Sci. USA 2002, 99, 11593-11598. [CrossRef]

11. Patel, D.J.; Phan, A.T.; Kuryavyi, V. Human telomere, oncogenic promoter and 5'-UTR G-quadruplexes: Diverse higher order DNA and RNA targets for cancer therapeutics. Nucleic Acids Res. 2007, 35, 7429-7455. [CrossRef] [PubMed]

12. Lipps, H.J.; Rhodes, D. G-quadruplex structures: In vivo evidence and function. Trends Cell Biol. 2009, 19, 414-422. [CrossRef] [PubMed]

13. Bugaut, A.; Balasubramanian, S. 5'-UTR RNA G-quadruplexes: Translation regulation and targeting. Nucleic Acids Res. 2012, 40, 4727-4741. [CrossRef] [PubMed]

14. Le, D.D.; Di Antonio, M.; Chan, L.K.; Balasubramanian, S. G-quadruplex ligands exhibit differential G-tetrad selectivity. Chem. Commun. 2015, 51, 8048-8050. [CrossRef] [PubMed]

15. Ranjan, N.; Davis, E.; Xue, L.; Arya, D.P. Dual recognition of the human telomeric G-quadruplex by a neomycin-anthraquinone conjugate. Chem. Commun. 2013, 49, 5796-5798. [CrossRef] [PubMed]

16. Wei, C.Y.; Jia, G.Q.; Zhou, J.; Han, G.Y.; Li, C. Evidence for the binding mode of porphyrins to G-quadruplex DNA. Phys. Chem. Chem. Phys. 2009, 11, 4025-4032. [CrossRef] [PubMed]

17. Phan, A.T.; Kuryavyi, V.; Gaw, H.Y.; Patel, D.J. Small-molecule interaction with a five-guanine-tract G-quadruplex structure from the human MYC promoter. Nat. Chem. Biol. 2005, 1, 167-173. [CrossRef]

18. Chung, W.J.; Heddi, B.; Hamon, F.; Teulade-Fichou, M.P.; Phan, A.T. Solution structure of a G-quadruplex bound to the bisquinolinium compound Phen-DC3. Angew. Chem. Int. Ed. 2014, 53, 999-1002. [CrossRef] 
19. Marchand, A.; Granzhan, A.; Iida, K.; Tsushima, Y.; Ma, Y.; Nagasawa, K.; Teulade-Fichou, M.P.; Gabelica, V. Ligand-Induced Conformational Changes with Cation Ejection upon Binding to Human Telomeric DNA G-Quadruplexes. J. Am. Chem. Soc. 2015, 137, 750-756. [CrossRef]

20. Hamon, F.; Largy, E.; Guedin-Beaurepaire, A.; Rouchon-Dagois, M.; Sidibe, A.; Monchaud, D.; Mergny, J.L.; Riou, J.F.; Nguyen, C.H.; Teulade-Fichou, M.P. An Acyclic Oligoheteroaryle That Discriminates Strongly between Diverse G-Quadruplex Topologies. Angew. Chem. Int. Ed. 2011, 50, 8745-8749. [CrossRef]

21. Martino, L.; Virno, A.; Pagano, B.; Virgilio, A.; Di Micco, S.; Galeone, A.; Giancola, C.; Bifulco, G.; Mayol, L.; Randazzo, A. Structural and thermodynamic studies of the interaction of distamycin A with the parallel quadruplex structure [d(TGGGGT)]4. J. Am. Chem. Soc. 2007, 129, 16048-16056. [CrossRef]

22. Iida, K.; Nagasawa, K. Macrocyclic Polyoxazoles as G-Quadruplex Ligands. Chem. Rec. 2013, 13, 539-548. [CrossRef]

23. Kim, M.Y.; Vankayalapati, H.; Shin-Ya, K.; Wierzba, K.; Hurley, L.H. Telomestatin, a potent telomerase inhibitor that interacts quite specifically with the human telomeric intramolecular g-quadruplex. J. Am. Chem. Soc. 2002, 124, 2098-2099. [CrossRef] [PubMed]

24. Shin-ya, K.; Wierzba, K.; Matsuo, K.; Ohtani, T.; Yamada, Y.; Furihata, K.; Hayakawa, Y.; Seto, H. Telomestatin, a novel telomerase inhibitor from Streptomyces anulatus. J. Am. Chem. Soc. 2001, 123, 1262-1263. [CrossRef]

25. Sakuma, M.; Ma, Y.; Tsushima, Y.; Iida, K.; Hirokawa, T.; Nagasawa, K. Design and synthesis of unsymmetric macrocyclic hexaoxazole compounds with an ability to induce distinct G-quadruplex topologies in telomeric DNA. Org. Biomol. Chem. 2016, 14, 5109-5116. [CrossRef] [PubMed]

26. Ma, Y.; Tsushima, Y.; Sakuma, M.; Sasaki, S.; Iida, K.; Okabe, S.; Seimiya, H.; Hirokawa, T.; Nagasawa, K. Development of G-quadruplex ligands for selective induction of a parallel-type topology. Org. Biomol. Chem. 2018, 16, 7375-7382. [CrossRef]

27. Teulade-Fichou, M.P.; Carrasco, C.; Guittat, L.; Bailly, C.; Alberti, P.; Mergny, J.L.; David, A.; Lehn, J.M.; Wilson, W.D. Selective recognition of G-qQuadruplex telomeric DNA by a bis(quinacridine) macrocycle. J. Am. Chem. Soc. 2003, 125, 4732-4740. [CrossRef] [PubMed]

28. Di Leva, F.S.; Zizza, P.; Cingolani, C.; D’Angelo, C.; Pagano, B.; Amato, J.; Salvati, E.; Sissi, C.; Pinato, O.; Marinelli, L.; et al. Exploring the chemical space of G-quadruplex binders: Discovery of a novel chemotype targeting the human telomeric sequence. J. Med. Chem. 2013, 56, 9646-9654. [CrossRef]

29. Maji, B.; Kumar, K.; Kaulage, M.; Muniyappa, K.; Bhattacharya, S. Design and synthesis of new benzimidazole-carbazole conjugates for the stabilization of human telomeric DNA, telomerase inhibition, and their selective action on cancer cells. J. Med. Chem. 2014, 57, 6973-6988. [CrossRef]

30. Lavrado, J.; Ohnmacht, S.A.; Correia, I.; Leitao, C.; Pisco, S.; Gunaratnam, M.; Moreira, R.; Neidle, S.; Santos, D.J.; Paulo, A. Indolo [3,2-c]quinoline G-quadruplex stabilizers: A structural analysis of binding to the human telomeric G-quadruplex. Chem. Med. Chem. 2015, 10, 836-849. [CrossRef]

31. Abraham Punnoose, J.; Ma, Y.; Li, Y.; Sakuma, M.; Mandal, S.; Nagasawa, K.; Mao, H. Adaptive and Specific Recognition of Telomeric G-Quadruplexes via Polyvalency Induced Unstacking of Binding Units. J. Am. Chem. Soc. 2017, 139, 7476-7484. [CrossRef] [PubMed]

32. Pennarun, G.; Granotier, C.; Gauthier, L.R.; Gomez, D.; Boussin, F.D. Apoptosis related to telomere instability and cell cycle alterations in human glioma cells treated by new highly selective G-quadruplex ligands. Oncogene 2005, 24, 2917-2928. [CrossRef] [PubMed]

33. Ruden, M.; Puri, N. Novel anticancer therapeutics targeting telomerase. Cancer Treat. Rev. 2013, 39, 444-456. [CrossRef] [PubMed]

34. $\mathrm{Xu}, \mathrm{Y}$. Chemistry in human telomere biology: Structure, function and targeting of telomere DNA/RNA. Chem. Soc. Rev. 2011, 40, 2719-2740. [CrossRef] [PubMed]

35. Balasubramanian, S.; Neidle, S. G-quadruplex nucleic acids as therapeutic targets. Curr. Opin. Chem. Biol. 2009, 13, 345-353. [CrossRef] [PubMed]

36. Hansel-Hertsch, R.; Di Antonio, M.; Balasubramanian, S. DNA G-quadruplexes in the human genome: Detection, functions and therapeutic potential. Nat. Rev. Mol. Cell Biol. 2017, 18, 279-284. [CrossRef] [PubMed]

37. Chen, S.B.; Hu, M.H.; Liu, G.C.; Wang, J.; Ou, T.M.; Gu, L.Q.; Huang, Z.S.; Tan, J.H. Visualization of NRAS RNA G-Quadruplex Structures in Cells with an Engineered Fluorogenic Hybridization Probe. J. Am. Chem. Soc. 2016, 138, 10382-10385. [CrossRef] 
38. Hu, M.H.; Chen, S.B.; Wang, B.; Ou, T.M.; Gu, L.Q.; Tan, J.H.; Huang, Z.S. Specific targeting of telomeric multimeric G-quadruplexes by a new triaryl-substituted imidazole. Nucleic Acids Res. 2017, 45, 1606-1618. [CrossRef]

39. Hu, M.H.; Wang, Y.Q.; Yu, Z.Y.; Hu, L.N.; Ou, T.M.; Chen, S.B.; Huang, Z.S.; Tan, J.H. Discovery of a New Four-Leaf Clover-Like Ligand as a Potent c-MYC Transcription Inhibitor Specifically Targeting the Promoter G-Quadruplex. J. Med. Chem. 2018, 61, 2447-2459. [CrossRef]

40. Chung, W.J.; Heddi, B.; Tera, M.; Iida, K.; Nagasawa, K.; Phan, A.T. Solution structure of an intramolecular $(3+1)$ human telomeric G-quadruplex bound to a telomestatin derivative. J. Am. Chem. Soc. 2013, 135, 13495-13501. [CrossRef]

41. Iida, K.; Majima, S.; Ohtake, T.; Tera, M.; Shin-ya, K.; Nagasawa, K. Design and synthesis of g-quadruplex ligands bearing macrocyclic hexaoxazoles with four-way side chains. Heterocycles 2012, 84, 401-411.

42. Tera, M.; Ishizuka, H.; Takagi, M.; Suganuma, M.; Shin-ya, K.; Nagasawa, K. Macrocyclic hexaoxazoles as sequence- and mode-selective G-quadruplex binders. Angew. Chem. Int. Ed. 2008, 47, 5557-5560. [CrossRef] [PubMed]

43. De Cian, A.; Guittat, L.; Kaiser, M.; Sacca, B.; Amrane, S.; Bourdoncle, A.; Alberti, P.; Teulade-Fichou, M.P.; Lacroix, L.; Mergny, J.L. Fluorescence-based melting assays for studying quadruplex ligands. Methods 2007, 42, 183-195. [CrossRef] [PubMed]

44. Ou, T.M.; Lu, Y.J.; Tan, J.H.; Huang, Z.S.; Wong, K.Y.; Gu, L.Q. G-quadruplexes: Targets in anticancer drug design. ChemMedChem 2008, 3, 690-713. [CrossRef] [PubMed]

45. Vorlickova, M.; Kejnovska, I.; Sagi, J.; Renciuk, D.; Bednarova, K.; Motlova, J.; Kypr, J. Circular dichroism and guanine quadruplexes. Methods 2012, 57, 64-75. [CrossRef] [PubMed]

46. The sterically hindered side chains introduced in the compounds $\mathbf{4}$ and $\mathbf{5}$ might cause the topology change from hybrid into parallel structure by interfering with the loops.

47. To evaluate the docking accuracy of our protocol, we carried out the re-docking study using the known x-ray structure of G-quadruplex-ligand complex (PDB ID: 3UYH). In the re-docking experiments, we obtained near native poses (RMSD < $3 \AA$ ) in top 100 candidates, however, correlation between the docking scores and the RMSD plots were low. To avoid the choices of the false positive poses from the best-rank only, we decided to consider 100 poses together with the docking scores. See Figure S2.

48. Friesner, R.A.; Banks, J.L.; Murphy, R.B.; Halgren, T.A.; Klicic, J.J.; Mainz, D.T.; Repasky, M.P.; Knoll, E.H.; Shelley, M.; Perry, J.K.; et al. Glide: A new approach for rapid, accurate docking and scoring. 1. Method and assessment of docking accuracy. J. Med. Chem. 2004, 47, 1739-1749. [CrossRef] [PubMed]

49. Halgren, T.A. Identifying and characterizing binding sites and assessing druggability. J. Chem. Inf. Model. 2009, 49, 377-389. [CrossRef]

Sample Availability: Samples of all compounds are available from the authors.

(C) 2019 by the authors. Licensee MDPI, Basel, Switzerland. This article is an open access article distributed under the terms and conditions of the Creative Commons Attribution (CC BY) license (http://creativecommons.org/licenses/by/4.0/). 